\title{
Multimodal Percutaneous Thermal Ablation of Small Hepatocellular Carcinoma: Predictive Factors of Recurrence and Survival in Western Patients
}

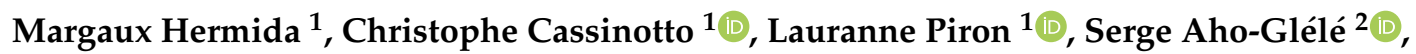 \\ Chloé Guillot ${ }^{1}$, Valentina Schembri ${ }^{1}$, Carole Allimant ${ }^{1}$, Samir Jaber ${ }^{3}$, \\ Georges-Philippe Pageaux ${ }^{4}$, Eric Assenat ${ }^{5}$ and Boris Guiu ${ }^{1, *}$ \\ 1 Department of Radiology, St-Eloi University Hospital, 34980 Montpellier, France; \\ m-hermida@chu-montpellier.fr (M.H.); c-cassinotto@chu-montpellier.fr (C.C.); \\ 1-piron@chu-montpellier.fr (L.P.); chloe-guillot@chu-montpellier.fr (C.G.); \\ v-schembri@chu-montpellier.fr (V.S.); carole.allimant@gmail.com (C.A.) \\ 2 Department of Epidemiology, Dijon University Hospital, 21000 Dijon, France; ludwig.aho@chu-dijon.fr \\ 3 Department of Anesthesiology and critical care, St-Eloi University Hospital, 34295 Montpellier, France; \\ s-jaber@chu-montpellier.fr \\ 4 Department of Hepatology, St-Eloi University Hospital, 34295 Montpellier, France; \\ gp-pageaux@chu-montpellier.fr \\ 5 Department of Oncology, St-Eloi University Hospital, 34295 Montpellier, France; \\ e-assenat@chu-montpellier.fr \\ * Correspondence: B-guiu@chu-montpellier.fr; Tel.: +33-467-336-733; Fax: +33-467-337-623
}

Received: 27 December 2019; Accepted: 26 January 2020; Published: 29 January 2020

\begin{abstract}
Background: To identify the predictive factors of recurrence and survival in an unselected population of Western patients who underwent multimodal percutaneous thermal ablation (PTA) for small Hepatocellular Carcinomas (HCCs). Methods: January 2015-June 2019: data on multimodal PTA for $<3 \mathrm{~cm}$ HCC were extracted from a prospective database. Local tumor progression (LTP), intrahepatic distant recurrence (IDR), time-to-LTP, time-to-IDR, recurrence-free (RFS) and overall (OS) survival were evaluated. Results: 238 patients underwent 317 PTA sessions to treat 412 HCCs. During follow-up (median: 27.1 months), $47.1 \%$ patients had IDR and $18.5 \%$ died. LTP occurred after $13.3 \%$ of PTA. Tumor size $(\mathrm{OR}=1.108, p<0.001$; hazard ratio $(\mathrm{HR})=1.075, p=0.002)$ and ultrasound guidance $(\mathrm{OR}=0.294, p=0.017 ; \mathrm{HR}=0.429, p=0.009)$ independently predicted LTP and time-to-LTP, respectively. Alpha fetoprotein $(\mathrm{AFP})>100 \mathrm{ng} / \mathrm{mL}(\mathrm{OR}=3.027, p=0.037)$ and tumor size $(\mathrm{OR}=1.06$, $p=0.001)$ independently predicted IDR. Multinodular HCC $(\mathrm{HR}=2.67, p<0.001)$, treatment-naïve patient $(\mathrm{HR}=0.507, p=0.002)$ and AFP $>100 \mathrm{ng} / \mathrm{mL}(\mathrm{HR}=2.767, p=0.014)$ independently predicted time-to-IDR. RFS was independently predicted by multinodular HCC $(\mathrm{HR}=2.144, p=0.001)$, treatment naivety $(\mathrm{HR}=0.546, p=0.004)$ and AFP $>100 \mathrm{ng} / \mathrm{mL}(\mathrm{HR}=2.437, p=0.013)$. The American Society of Anesthesiologists (ASA) score $>2(\mathrm{HR}=4.273, p=0.011)$, AFP $(\mathrm{HR}=1.002, p<0.001)$, multinodular HCC $(\mathrm{HR}=3.939, p=0.003)$ and steatotic HCC $\left(\mathrm{HR}=1.81 \times 10^{-16}, p<0.001\right)$ independently predicted OS. Conclusions: IDR was associated with tumor aggressiveness, suggesting a metastatic mechanism. Besides AFP association with LTP, IDR, RFS and OS, treatment-naïve patients had longer RFS, and multi-nodularity was associated with shorter RFS and OS. Steatotic HCC, identified on pre-treatment MRI, independently predicted longer OS, and needs to be further explored.
\end{abstract}

Keywords: hepatocellular carcinoma; percutaneous thermal ablation; recurrence; survival; liver; radiofrequency ablation; microwave ablation 


\section{Introduction}

HCC is the fifth most common cancer, and the third leading cause of death from cancer worldwide [1]. Percutaneous thermal ablation (PTA) is a validated treatment option for very early and early stage HCC, together with surgical resection and liver transplantation [2]. Ultrasonography provides real-time guidance, and for that reason is the main or even the only guidance modality [3-8] for PTA of HCC. However, about $50 \%$ of planned PTAs are considered unfeasible due to invisible/inconspicuous tumor, or unsafe needle path during the planning ultrasonography [3]. Consequently, $30 \%$ of patients referred for PTA are eventually treated with palliative therapies [3], a phenomenon also noticed in real-life studies, where about $35 \%$ of patients with early HCC receive suboptimal palliative rather than curative treatments [9-11]. Moreover, PTA is frequently not performed in the case of high-risk HCC location (liver dome, subcapsular, near a large vessel or an adjacent organ) [12]. All of these technical issues could lead to considerable selection bias in the routine practice, thus explaining the choice of palliative treatments in many cases. Since several years, multimodality interventional suites have become available to better visualize the tumor and to allow PTA of HCC in challenging locations through the use of ultrasonography, computed tomography (CT) and angiography, alone or in combination (i.e., multimodal PTA). However, few data are available on the outcomes of multimodal PTA in unselected populations of patients with small HCC.

The risk of local tumor progression (LTP) $(10 \%-30 \%)$ is one major concern of PTA [10]. This wide range may be explained by the considerable variety of PTA indications, techniques and imaging guidance modalities. Whether tumor size $>3 \mathrm{~cm}$ has been identified as a strong LTP predictor after monopolar PTA [2], some debate has emerged regarding 2-3 cm tumors [13]. Moreover, the long-term results of PTA are influenced by the high rate (up to $60 \%-80 \%$ at 5 years) of intrahepatic distant recurrence (IDR) $[7,10,14]$, as observed also after HCC surgical resection [2,15]. It is unknown whether early IDR ( $2-3$ years after PTA of $<3 \mathrm{~cm} \mathrm{HCC}$ ) is due to metastatic spread or de novo carcinogenesis. This is a crucial issue, particularly for developing neoadjuvant and/or adjuvant treatments, but proves difficult to assess, because basically, tumor specimens are rarely available after PTA of the primary HCC and also of the IDRs, which are frequently treated by PTA as well [16].

Therefore, the purpose of this study was to summarize the results of multimodal PTA and to identify some predictive factors of recurrence and survival in Western patients treated by multimodal PTA for small HCC.

\section{Materials and Methods}

\subsection{Patients}

This retrospective analysis was performed using data prospectively collected in a database of patients who underwent percutaneous thermal ablation (PTA) for Hepatocellular Carcinoma (HCC) at our institution. This study was approved by the Local Ethics and Research Committee (authorization number: 2017_CLER-MTP_12-04, NCT03428321 (www.clinicaltrials.gov)), and written, informed consent for the procedure and the prospective anonymized data collection was obtained from all patients during the consultation for PTA.

Inclusion criteria were: HCC diagnosed by histopathology analysis or by imaging, according to the European Association for the Study of the Liver (EASL) guidelines (23); HCC nodule(s) without extrahepatic metastases or macrovascular invasion; tumor size $\leq 30 \mathrm{~mm}$; World Health Organization (WHO) performance status 0 or 1 ; prothrombin time (PT) ratio $>50 \%$ and platelet count higher than $5 \times 10^{9} / \mathrm{L}$.

Exclusion criteria were: follow-up $<2$ months; Child-Pugh class B cirrhosis with a score $>7$; HCC nodule adjacent to the hepatic hilum; history of biliary-digestive anastomosis or endoscopic sphincterotomy; combined treatment with embolization or chemoembolization.

Treatment was validated at a multidisciplinary meeting that included interventional radiologists, liver surgeons, oncologists, hepatologists and radiation oncologists. PTA was considered as the first-line 
treatment for patients with HCC stage 0 (very early stage) and stage A (early stage) according to the Barcelona Clinic Liver Cancer (BCLC) staging system [2], meaning that no technical contraindication was considered, except HCC nodule adjacent hepatic hilum.

All patients underwent contrast-enhanced multiphase computed tomography (CT) and/or magnetic resonance imaging (MRI) (including dynamic MRI) within 1 month before PTA. Laboratory tests were systematically performed to detect any possible PTA contraindication. Then, all patients were seen in consultation by the interventional radiologist.

\section{Patient and Tumor Data}

The following patient data were collected: age, sex, American Society of Anesthesiologists (ASA) physical status score (assessed by the anesthesiologist) (24), diabetes mellitus, liver disease (cirrhosis was defined as typical hepatic dysmorphia on imaging or by histological analysis of liver biopsy; patients were considered non-cirrhotic if their liver was defined as healthy at histology; the others were considered undetermined), steatosis (defined as signal intensity loss on opposed-phase gradient-echo sequences at baseline or at the first follow-up MRI), and Child-Pugh, MELD and ALBI scores.

The following HCC characteristics were also collected: intra-tumor steatosis (defined as a signal intensity loss on opposed-phase gradient-echo sequences) (Figure S1), dome location (i.e., when the lung parenchyma is interposed between the skin and the tumor through the anterior or lateral route on the axial plane on the baseline CT or MRI [17]), subcapsular location (i.e., direct contact with the liver capsule), adjacent to large vessels (i.e., located $\leq 5 \mathrm{~mm}$ from any $\geq 3 \mathrm{~mm}$ vessel) (29), adjacent to at-risk organs (i.e., located $\leq 5 \mathrm{~mm}$ from the right kidney, adrenal gland, stomach, gut or colon).

\subsection{Percutaneous Thermal Ablation}

Patients were placed in a supine position with arms above the head when possible. All procedures were done under general anesthesia with endotracheal intubation. Patients were mechanically ventilated using low tidal volume ventilation (i.e., tidal volume between 3 to $4 \mathrm{~mL} / \mathrm{kg}, 320 \mathrm{~mL} / \mathrm{min}$ minimum) to strongly limit liver movements. Respiratory rate was adjusted to maintain the end tidal carbon dioxide between 35 and $45 \mathrm{mmHg}$.

Guidance and Ablation

All PTAs were performed in a multimodality interventional suite. The suite was equipped with a CT scan (Optima 660, General Electric, Milwaukee, Brookfield, WI, USA) with a combined mobile C-arm (OE9900, General Electric, Milwaukee) from January 2015 to February 2017, and then with an Angio-CT Infinix-I 4D CT system (Canon Medical Systems, Tokyo, Japan) from February 2017. An ultrasound machine (Logiq E9, General Electric, Milwaukee) was available during the entire study period.

PTA was performed by four interventional radiologists (5-15 years of expertise in liver PTA) using a radiofrequency or a microwave device, depending on the operator's choice. Radiofrequency ablations (RFAs) were performed using a clustered internally-cooled (Covidien E series, Covidien, Boulder, CO, USA) or a separable clustered internally-cooled (OctopusR, STARmed, Goyang, Korea) electrode. For this second system, one, two or three electrodes could be used. When two or three electrodes were needed, the generator (VIVA MultiR, STARmed) was in dual-switch mode. Microwave ablations (MWAs) were performed using a single 15G internally-cooled electrode and the Acculis MTA system (Angiodynamics, Amsterdam, The Netherlands). Whatever the technique, the objective was to completely ablate the tumor(s) with a $5 \mathrm{~mm}-1 \mathrm{~cm}$ margin. Needle track ablation was performed according to the manufacturer's instructions.

Ultrasonography (US) guidance was the first-line guidance modality, i.e., when the tumor was visible using US, PTA was performed using US guidance. When the tumor was not visible using US or unenhanced $\mathrm{CT}$, the tumor was tagged. Specifically, an angiographic catheter (4F or $5 \mathrm{~F})$ was inserted in the celiac trunk or the superior mesenteric artery through the femoral route. Then, a 2.7F micro-catheter (Progreat, Terumo, Tokyo, Japan) was used to reach the right or left hepatic artery (depending on the anatomical variants and HCC location). 
After injection of 1.5-2 mL of lipiodol (Lipiodol ultrafluide, Guerbet, Aulnay-sous-bois, France), tumor tagging was confirmed by immediate post-injection CT. Then, CT-fluoroscopy was used to guide the PTA needle insertion in the tumor(s) (Figure S2). For tumors that are located in the liver dome and are invisible by ultrasonography, the extrapulmonary transthoracic transdiaphragmatic route was used after artificially-induced pneumothorax with $\mathrm{CO}_{2}$, as previously described $[17,18]$ (Figure S3).

Hydro (5\% dextrose) or $\mathrm{CO}_{2}$ dissection was used whenever necessary to protect the surrounding organs against thermal injury [19]. Intermittent balloon occlusion of the hepatic vein [20] was used when necessary to prevent the heat sink effect (Figure S4).

The following technical factors were systematically recorded: PTA modality (RFA or MWA), imaging guidance (ultrasonography or $\mathrm{CT}$ ), artificial pneumothorax and tumor tagging.

Contrast-enhanced CT (portal phase) was performed immediately after the procedure to evaluate the ablation zone (i.e., the area of low attenuation) and to detect post-procedural complications. In the case of incomplete ablation or insufficient margins, the ablation needle(s) was (were) re-inserted during the same procedure to achieve complete ablation.

\subsection{Complications}

The type and number of complications were recorded and classified according to the Society of Interventional Radiology (SIR) guidelines [21]. Patients were monitored overnight. All patients underwent a clinical examination and laboratory tests the day after PTA.

\subsection{Follow-Up and Outcome}

"Technical success" was defined as complete ablation of the target tumor(s) [21] on the immediate post-PTA CT images.

Patients were followed by the same interventional radiologist who performed the PTA in consultation, immediately after the first follow-up MRI (including dynamic acquisitions) performed 4-6 weeks after the procedure, and then every 3 months.

"Treatment failure" was defined as the presence of persistent enhanced foci at the tumor site on the first follow-up MRI [22].

Complete ablation observed on first follow-up MRI was considered as the "primary treatment success". "Secondary treatment success" was treatment success observed only after a second PTA performed within 8 weeks after the first one.

"Local tumor progression" (LTP) was any growing or enhanced tumor focus within or at the edge (direct contact) of the ablation zone, after complete ablation documented by at least one MRI [22].

"Intrahepatic distant recurrence" (IDR) was defined as any new HCC nodule in the liver, defined according to the EASL criteria [22].

\subsection{Statistical Analysis}

Normally distributed continuous variables were described with mean $\pm S D$, non-normally distributed continuous variables with median/interquartile range (IQR), and categorical variables with numbers/percentages. Categorical variables were compared with the Fischer's exact test, and continuous variables with the two-sided t-test or Kruskal-Wallis test, as appropriate.

The association between LTP or IDR and all patient, liver, tumor and PTA characteristics was assessed using univariate and multivariate logistic regression analyses to compute the odds ratio (OR) (with 95\% confidence intervals (95\% CIs)). In all cases, repeated measures logistic regression analyses were performed using a generalized estimating equation (GEE) logistic regression model to take into account PTA of several nodules and repeated PTA sessions. A robust variance estimator was used systematically. Log-linearity was checked using fractional polynomials. For the GEE model, an exchangeable correlation matrix was chosen and checked using quasi-likelihood information criteria [23].

Time-to-LTP and time-to-IDR were defined as the time from PTA to LTP and to IDR, respectively. Patients without LTP/IDR at the last follow-up/death/liver transplantation were censored at the date of the last follow-up/death/liver transplantation. 
RFS was defined as the interval between PTA and death, recurrence, or last follow-up. OS was defined as the interval between PTA and death or last follow-up. Patients who underwent liver transplantation were censored at the transplantation date.

The median follow-up (95\% CI) was calculated using the reverse Kaplan-Meier method. Survival curves were estimated using the Kaplan-Meier method and compared using the log-rank test. Univariate Cox proportional-hazards models of all potential baseline predictors were built to compute the hazard ratios (HRs) with their 95\% CIs. Multivariate Cox models were built to include all variables found to be significant in the univariate analyses. Bootstrapping was systematically performed for internal validation (400 replications for per-tumor analyses, 200 replications for per-patient analyses).

All analyses were performed with the Stata software, version 14.0 (Stata corporation, College Station, TX, USA). A $p$-value $<0.05$ was considered significant.

\section{Results}

\subsection{Patient Characteristics}

Between January 2015 and June 2019, 255 consecutive patients underwent PTA for HCC at our institution. Seventeen patients were excluded due to combined treatment with embolization $(n=9)$, metastatic progression discovered at PTA day $(n=1), \mathrm{HCC}>30 \mathrm{~mm}(n=4)$ and follow-up $<2$ month $(n=3)$. Finally, 238 patients (median age: $64.9 \pm 9.9$ years; $79.8 \%(190 / 238)$ men) who underwent 317 PTA sessions to treat 412 HCCs were included in this study (Figure 1, and Table 1 for patient, liver disease, HCC characteristics and PTA technical factors).

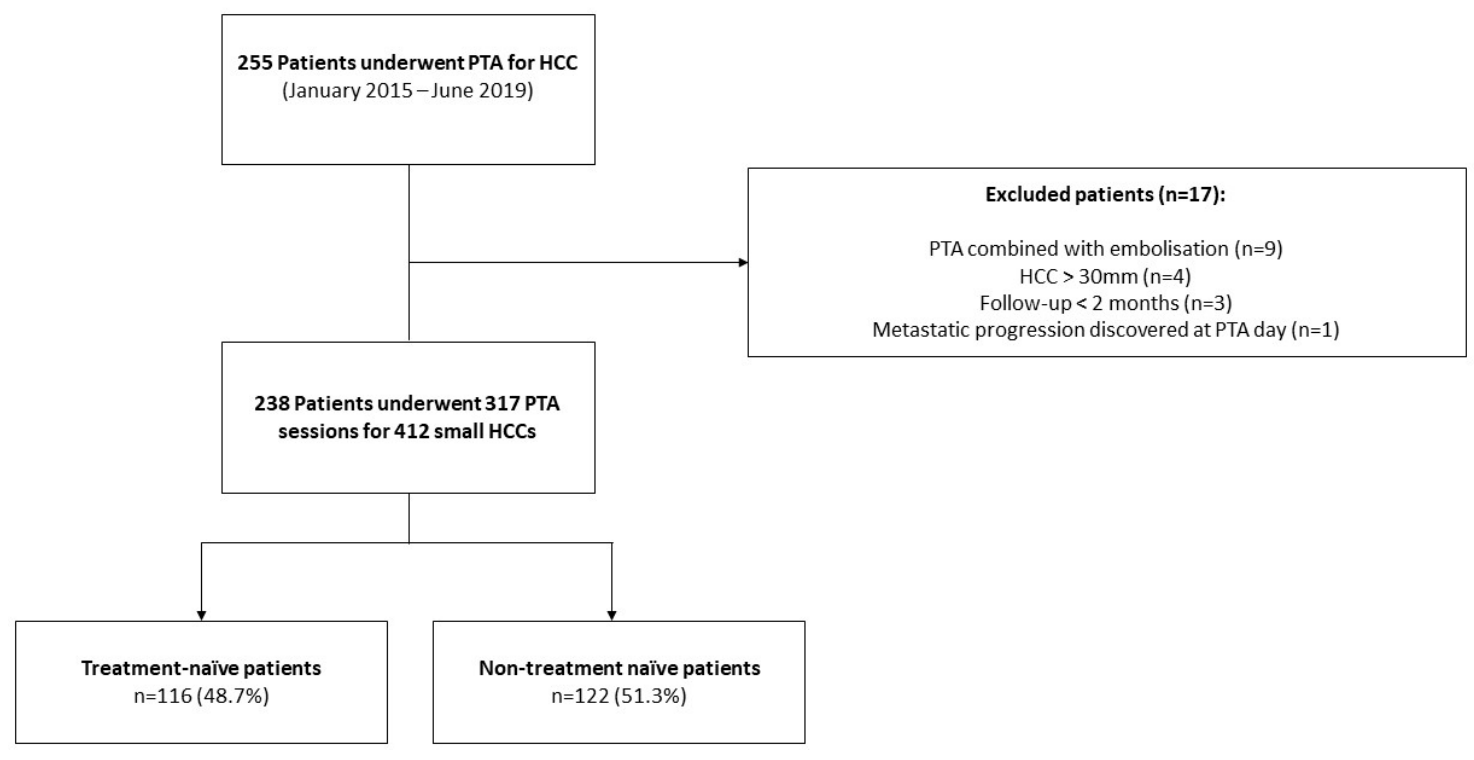

Figure 1. Study Flowchart.

Table 1. Characteristics of the 238 patients with 412 small Hepatocellular Carcinomas (HCCs) treated by percutaneous thermal ablation (PTA).

\begin{tabular}{|c|c|}
\hline Characteristic & Value \\
\hline \multicolumn{2}{|c|}{ Patients } \\
\hline Age (mean $\pm S D$, years) & $64.9 \pm 9.9$ \\
\hline \multicolumn{2}{|l|}{$\operatorname{Sex}(n, \%)$} \\
\hline Male & $190(79.8)$ \\
\hline Female & $48(20.29)$ \\
\hline \multicolumn{2}{|l|}{ ASA score $(n, \%)$} \\
\hline $1-2$ & $120(50.4)$ \\
\hline $3-4$ & $118(49.6)$ \\
\hline
\end{tabular}


Table 1. Cont.

\begin{tabular}{|c|c|}
\hline Characteristic & Value \\
\hline \multicolumn{2}{|c|}{ Patients } \\
\hline \multicolumn{2}{|l|}{ Diabetes $(n, \%)$} \\
\hline No & $147(61.8)$ \\
\hline Yes & $91(38.2)$ \\
\hline Metformin treatment & $35(14.7)$ \\
\hline \multicolumn{2}{|l|}{ Prior treatment for $\operatorname{HCC}(n, \%)$} \\
\hline No & $116(48.7)$ \\
\hline Yes & $122(51.3)$ \\
\hline Resection & $33(27)$ \\
\hline PTA & $55(45)$ \\
\hline TACE & $34(28)$ \\
\hline \multicolumn{2}{|c|}{ Liver disease } \\
\hline \multicolumn{2}{|l|}{ Cirrhosis $(n, \%)$} \\
\hline No & $20(8.4)$ \\
\hline Yes & $218(91.6$ \\
\hline \multicolumn{2}{|l|}{ Causes for liver disease $(n, \%)$} \\
\hline Alcohol (with or without viral hepatitis) & $135(56.7)$ \\
\hline Viral hepatitis B or C & $61(25.6)$ \\
\hline Hemochromatosis & $12(5)$ \\
\hline Other causes, including NASH & $30(12.6)$ \\
\hline \multicolumn{2}{|l|}{ Steatosis $(n, \%)$} \\
\hline No & $153(65.7)$ \\
\hline Yes & $80(34.3)$ \\
\hline \multicolumn{2}{|l|}{ Child-Pugh class } \\
\hline A & $231(97.1)$ \\
\hline $\mathrm{B}$ & $7(2.9)$ \\
\hline MELD score (mean \pm SD) & $8.8 \pm 2.2$ \\
\hline \multicolumn{2}{|l|}{ Laboratory data $($ mean \pm SD) } \\
\hline AFP (ng/mL) & $36.1 \pm 163.7$ \\
\hline Total bilirubin $(\mu \mathrm{mol} / \mathrm{L})$ & $13.5 \pm 8.7$ \\
\hline Albumin $(\mathrm{g} / \mathrm{L})$ & $40.2 \pm 4.9$ \\
\hline Prothrombin activity (\%) & $84.6 \pm 13.2$ \\
\hline Platelet count $\left(\times 10 / \mathrm{mm}^{3}\right)$ & $139 \pm 72$ \\
\hline Creatinine $(\mu \mathrm{mol} / \mathrm{L})$ & $83.7 \pm 31.1$ \\
\hline \multicolumn{2}{|l|}{ ALBI score } \\
\hline 1 & $151(66.8)$ \\
\hline 2 & $75(33.2)$ \\
\hline \multicolumn{2}{|l|}{ HCC } \\
\hline \multicolumn{2}{|l|}{ Method for diagnosis $(n, \%)$} \\
\hline Biopsy & $62(15)$ \\
\hline Imaging & $350(85)$ \\
\hline Tumor size (mean $\pm \mathrm{SD})$ & $15.1 \pm 5$ \\
\hline$<20 \mathrm{~mm}$ & $358(86.9)$ \\
\hline$>20 \mathrm{~mm}$ & $54(13.1)$ \\
\hline \multicolumn{2}{|l|}{ No. of tumors $(n, \%)$} \\
\hline 1 & $242(72.9)$ \\
\hline 2 & $56(21.4)$ \\
\hline 3 & $18(5.4)$ \\
\hline 4 & $1(0.3)$ \\
\hline
\end{tabular}


Table 1. Cont.

\begin{tabular}{lc}
\multicolumn{1}{c}{ Characteristic } & Value \\
\hline & HCC \\
Steatotic HCC $(n, \%)$ & \\
$\quad$ Yes & $66(16)$ \\
No & $317(77)$ \\
Unknown & $29(7)$ \\
Dome $(n, \%)$ & $93(22.6)$ \\
Subcapsular location $(n, \%)$ & $150(36.4)$ \\
Near large vessel $(n, \%)$ & $71(17.2)$ \\
Near surrounding organ $(n, \%)$ & $36(8.7)$ \\
\hline & \\
PTA modality $(n, \%)$ & \\
$\quad$ Radiofrequency & $174(42.2)$ \\
$\quad$ Microwave & $238(57.8)$ \\
\hline Imaging guidance $(n, \%)$ & \\
$\quad$ US & $182(44.2)$ \\
CT & $230(55.8)$ \\
Artificial CO ${ }_{2}$ pneumothorax $(n, \%)$ & $47(11.4)$ \\
Hydrodissection-CO ${ }_{2}$ dissection $(n, \%)$ & $36(8.7)$ \\
Tumor tagging $(n, \%)$ & $208(50.5)$ \\
\hline
\end{tabular}

Unless otherwise indicated, results are numbers (percentages). Abbreviations: SD, standard deviation; HCC, hepatocellular carcinoma; NASH, non-alcoholic steatohepatitis; MELD, model for end-stage liver disease; AFP, alpha fetoprotein; PTA, percutaneous thermal ablation; US, ultrasonography.

\subsection{Technical/Treatment Success}

Technical success was $100 \%$. Primary treatment success was obtained for $98.8 \%$ of nodules (407/412 HCCs). The five patients with primary treatment failure underwent a new and successful PTA procedure $(100 \%$ secondary treatment success).

\subsection{Complications and Follow-Up}

In total, 15 complications (3.6\%; 9/245) were observed (Table S2). No PTA-related death, needle track seeding, or liver abscess was reported. Patients were discharged the day after PTA in $94.6 \%$ (307/317) of cases.

During a median follow-up of 27.1 months (95\% CI: 23.2-29.8), 18.5\% (44/238) patients died and $9.2 \%(22 / 238)$ underwent liver transplantation.

\subsection{Local Tumor Progression}

LTP occurred after 55/412 (13.3\%) ablations. The 1-year, 2-year and 3-year cumulative LTP incidence (per-tumor analysis) were $8.7 \%, 16.6 \%$ and $21 \%$, respectively. The median time-to-LTP was 9.6 months (95\% CI: 1.8-30). LTP occurred before IDR in 50.9\% (28/55), after IDR in 20\% (11/55), and concomitantly with IDR in $29.1 \%(16 / 55)$ of cases.

The 55 LTP were treated with PTA $(n=42 ; 76.4 \%)$, super-selective, transarterial chemoembolization (TACE) $(n=8,14.5 \%)$, percutaneous ethanol injection $(n=1,1.8 \%)$, stereotactic body radiation therapy (SBRT) $(n=2,3.6 \%)$, or sorafenib $(n=1,1.8 \%)$.

In univariate analysis, age, tumor size, ultrasonography (vs. CT) guidance and tumor tagging were significantly associated with LTP (Table 2). In multivariate analysis, only tumor size (OR $=1.108$, $p<0.001$ ) and ultrasonography (vs. CT) guidance (OR $=0.294, p=0.017$ ) remained associated with LTP (Table 2). These results were internally validated using bootstrapping. Similarly, tumor size (HR $=1.075$, $p=0.002)$ and ultrasonography (vs. CT) guidance $(\mathrm{HR}=0.429, p=0.009)$ independently predicted the time-to-LTP (multivariate analysis, confirmed by internal validation using bootstrapping, Data not shown) (Figure 2). 
Table 2. Logistic regression analysis (generalized estimating equation (GEE) model) to predict local tumor progression (per tumor analysis).

\begin{tabular}{|c|c|c|c|c|c|c|}
\hline \multirow{2}{*}{ Variables } & \multicolumn{2}{|c|}{ Univariate Analysis } & \multicolumn{2}{|c|}{ Multivariate Analysis } & \multicolumn{2}{|c|}{ Bootstrapping $x(400$ Replications $)$} \\
\hline & Odds Ratio (95\% CI) & $p$ Value & Odds Ratio $(95 \% \mathrm{CI})$ & $p$ Value & Odds Ratio $(95 \%$ CI) & $p$ Value \\
\hline \multicolumn{7}{|c|}{ Patients } \\
\hline Age & $0.968(0.939-0.999)$ & 0.04 & $0.969(0.938-1.001)$ & 0.054 & $0.969(0.938-1.001)$ & 0.058 \\
\hline Sex female vs male & $0.487(0.189-1.252)$ & 0.136 & & & & \\
\hline ASA (>2 vs. $\leq 2)$ & $1.021(0.565-1.845)$ & 0.944 & & & & \\
\hline Diabetes & $1.665(0.904-3.066)$ & 0.102 & & & & \\
\hline Metformin treatment & $1.58(0.6-4.19)$ & 0.36 & & & & \\
\hline Treatment-naïve patient & $1.309(0.724-2.367)$ & 0.372 & & & & \\
\hline \multicolumn{7}{|c|}{ Liver diseases } \\
\hline Cirrhosis & $4.785(0.574-39.91)$ & 0.148 & & & & \\
\hline Child-Pugh (B vs. A) & $1.559(0.285-8.528)$ & 0.609 & & & & \\
\hline \multicolumn{7}{|l|}{ Cause of liver disease (vs. alcohol) } \\
\hline Viral hepatitis B or C & $0.624(0.279-1.392)$ & 0.249 & & & & \\
\hline Hemochromatosis & $0.92(0.273-3.097)$ & 0.893 & & & & \\
\hline Others (including NASH) & $0.859(0.325-2.268)$ & 0.758 & & & & \\
\hline Steatosis & $1.12(0.607-2.064)$ & 0.717 & & & & \\
\hline $\mathrm{AFP} \geq 100 \mathrm{vs}<100 \mathrm{ng} / \mathrm{mL}$ & $1.113(0.298-4.162)$ & 0.873 & & & & \\
\hline AFP (per unit) & $0.999(0.995-1.003)$ & 0.55 & & & & \\
\hline Bilirubin & $1.008(0.977-1.04)$ & 0.616 & & & & \\
\hline Albumin & $0.969(0.909-1.032)$ & 0.33 & & & & \\
\hline Prothrombin time & $1.001(0.979-1.024)$ & 0.922 & & & & \\
\hline 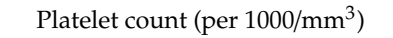 & $1(0.996-1.005)$ & 0.836 & & & & \\
\hline Creatinine & $0.997(0.985-1.008)$ & 0.552 & & & & \\
\hline $\operatorname{MELD~(>9~vs.~} \leq 9)$ & $1.263(0.69-2.312)$ & 0.449 & & & & \\
\hline ALBI score 2 vs. 1 & $1.241(0.65-2.37)$ & 0.512 & & & & \\
\hline \multicolumn{7}{|c|}{ HCC } \\
\hline Tumor size (per mm) & $1.096(1.04-1.154)$ & 0.001 & $1.108(1.05-1.169)$ & $<0.001$ & $1.108(1.051-1.168)$ & $<0.001$ \\
\hline Tumor size $<20 \mathrm{~mm}$ & $0.359(0.181-0.709)$ & 0.003 & & & & \\
\hline Steatotic HCC & $0.487(0.188-1.266)$ & 0.14 & & & & \\
\hline Dome tumor & $1.747(0.942-3.24)$ & .077 & & & & \\
\hline Subcapsular & $0.932(0.516-1.682)$ & 0.814 & & & & \\
\hline Near large vessel & $1.6096(0.809-3.202)$ & 0.175 & & & & \\
\hline Near surrounding organ & $1.043(0.387-2.809)$ & 0.934 & & & & \\
\hline \multicolumn{7}{|c|}{ PTA } \\
\hline PTA modality: MWA vs RF & $1.48(0.81-2.703)$ & 0.202 & & & & \\
\hline US vs CT guidance & $0.394(0.208-0.749)$ & 0.004 & $0.294(0.107-0.803)$ & 0.017 & $0.294(0.102-0.841)$ & 0.023 \\
\hline Artificial pneumothorax & $1.301(0.569-2.977)$ & 0.533 & & & & \\
\hline Tumor tagging & $1.82(1.01-3.28)$ & 0.046 & $0.827(0.325-2.103)$ & 0.689 & $0.827(0.362-1.887)$ & 0.651 \\
\hline
\end{tabular}

Abbreviations: HCC, hepatocellular carcinoma; NASH, non-alcoholic steatohepatitis; MELD, model for end-stage liver disease; AFP, alpha fetoprotein; PTA, percutaneous thermal ablation;

US, ultrasonography; CT, computed tomography. Significant $p$ values are marked in bold. 


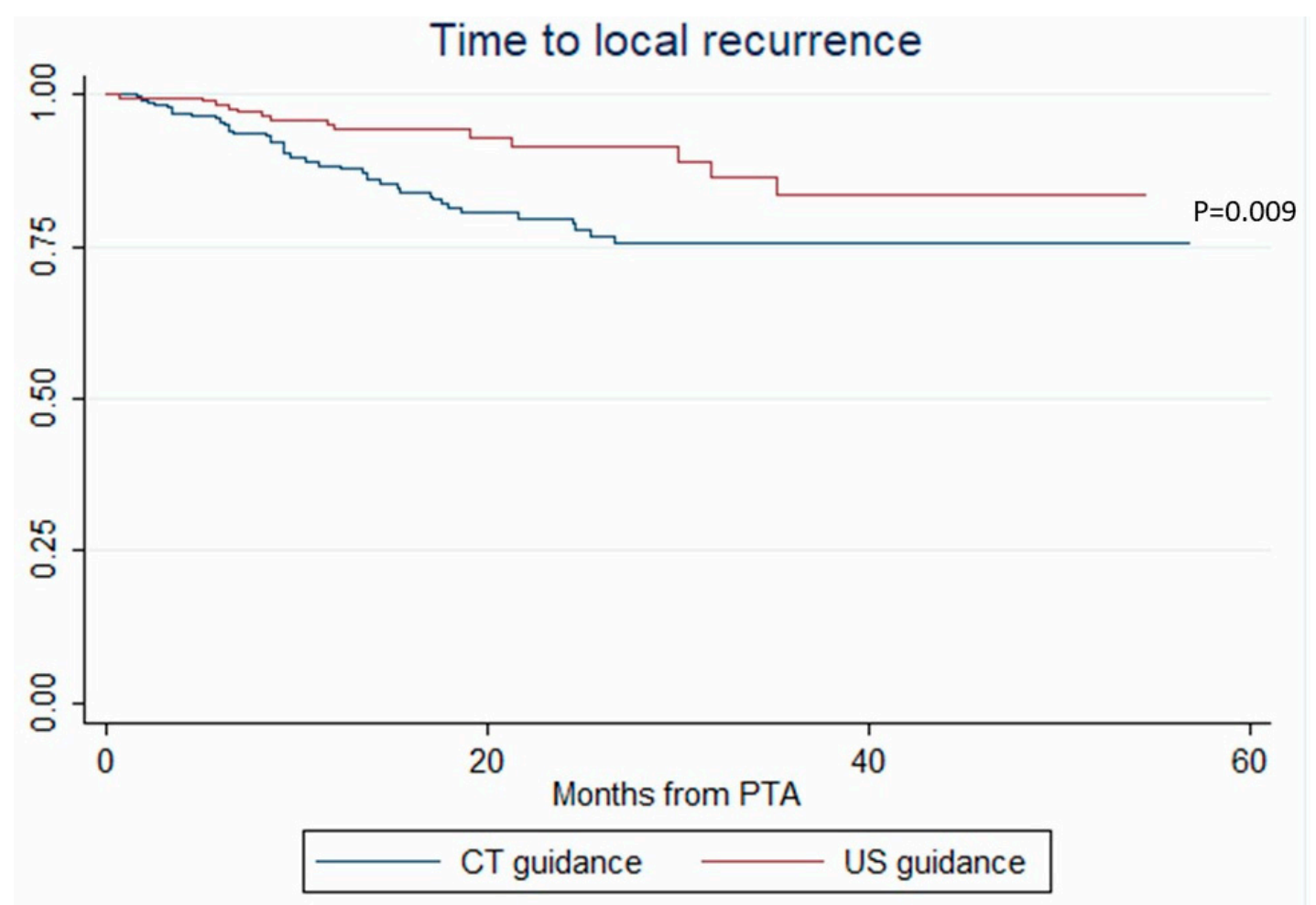

Figure 2. Time to local recurrence after percutaneous thermal ablation (PTA) under computed tomography (CT) and ultrasonography (US) guidance (Kaplan-Meier analysis).

\subsection{Intrahepatic Distant Recurrence}

IDR occurred in 112/238 (47.1\%) patients. The 1-year, 2-year and 3-year cumulative IRD incidences (per-patient analysis) were $33.3 \%, 46.9 \%$ and $63 \%$, respectively. The median time-to-IDR was 8.4 months (95\% CI: 1.6-34.3). IDR was treated with PTA $(n=55 ; 49.1 \%)$, super-selective TACE $(n=23,20.5 \%)$, sorafenib $(n=12,10.7 \%)$, percutaneous ethanol injection $(n=6 ; 5.4 \%)$, resection $(n=5 ; 4.5 \%)$, SBRT $(n=5 ; 4.5 \%)$, selective internal radiation therapy $(n=4 ; 3.6 \%)$ and best supportive care $(n=2 ; 1.8 \%)$.

The factors associated with IDR in univariate analysis are listed in Table 3 . In multivariate analysis, alpha fetoprotein (AFP) $>100 \mathrm{ng} / \mathrm{mL}(\mathrm{OR}=3.027 ; p=0.037)$ and tumor size $(\mathrm{OR}=1.06 ; p=0.001)$ were independently associated with IDR (internal validation by bootstrapping).

In multivariate (per-patient) analysis, multinodular HCC (HR $=2.67, p<0.001)$, treatment-naïve patient $(\mathrm{HR}=0.507, p=0.002)$ and AFP $>100 \mathrm{ng} / \mathrm{mL}(\mathrm{OR}=2.767 ; p=0.014)$ independently predicted time-to-IDR (confirmed after internal validation by bootstrapping, data not shown) (Figure 3). The median time-to-IDR was 40.4 vs. 18.2 months in treatment-naïve vs. non-treatment naïve patients $(p=0.001), 29.3$ vs. 5.1 months in patients with AFP $<100 \mathrm{ng} / \mathrm{mL}$ vs. AFP $\geq 100 \mathrm{ng} / \mathrm{mL}(p=0.002)$ and 32.4 vs. 8.4 months in patients with multinodular HCC vs. uninodular HCC $(p<0.001)$. 
Table 3. Logistic regression analysis (GEE model) to predict intrahepatic distant tumor progression (per-tumor analysis).

\begin{tabular}{|c|c|c|c|c|c|c|}
\hline \multirow{2}{*}{ Variables } & \multicolumn{2}{|c|}{ Univariate Analysis } & \multicolumn{2}{|c|}{ Multivariate Analysis } & \multicolumn{2}{|c|}{ Bootstrapping (400 Replications) } \\
\hline & Odds Ratio $(95 \% \mathrm{CI})$ & $p$ Value & Odds Ratio (95\% CI) & $p$ Value & Odds Ratio (95\% CI) & $p$ Value \\
\hline \multicolumn{7}{|c|}{ Patients } \\
\hline Age & $0.98(0.957-1.005)$ & 0.112 & & & & \\
\hline Sex female vs male & $0.953(0.526-1.727)$ & 0.874 & & & & \\
\hline ASA (>2 vs. $\leq 2)$ & $0.711(0.475-1.065)$ & 0.098 & & & & \\
\hline Diabetes & $0.668(0.394-1.131)$ & 0.133 & & & & \\
\hline Metformin treatment & $0.557(0.275-1.127)$ & 0.104 & & & & \\
\hline Treatment-naïve patient & $0.999(0.681-1.465)$ & 0.994 & & & & \\
\hline \multicolumn{7}{|c|}{ Liver diseases } \\
\hline Cirrhosis & $1.129(0.464-2.748)$ & 0.798 & & & & \\
\hline Child-Pugh (B vs. A) & $2.514(0.668-9.459)$ & 0.173 & & & & \\
\hline \multicolumn{7}{|l|}{ Cause of liver disease (vs. alcohol) } \\
\hline Viral hepatitis B or C & $1.067(0.605-1.88)$ & 0.823 & & & & \\
\hline Hemochromatosis & $0.955(0.321-2.847)$ & 0.934 & & & & \\
\hline Others (including NASH) & $0.753(0.351-1.618)$ & 0.468 & & & & \\
\hline Steatosis & $1.205(0.76-1.91)$ & 0.428 & & & & \\
\hline $\mathrm{AFP} \geq 100 \mathrm{vs}<100 \mathrm{ng} / \mathrm{mL}$ & $3.576(1.224-10.441)$ & 0.02 & $3.027(1.068-8.576)$ & 0.037 & $3.027(1.032-8.876)$ & 0.044 \\
\hline AFP (per unit) & $1.001(0.999-1.002)$ & 0.396 & & & & \\
\hline Bilirubin & $0.993(0.968-1.018)$ & 0.581 & & & & \\
\hline Albumin & $0.955(0.914-0.998)$ & 0.04 & $0.966(0.923-1.011)$ & 0.139 & $0.966(0.896-1.042)$ & 0.37 \\
\hline Prothrombin time & $0.996(0.98-1.012)$ & 0.668 & & & & \\
\hline 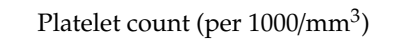 & $1(0.997-1.003)$ & 0.952 & & & & \\
\hline Creatinine & $0.992(0.984-1.002)$ & 0.087 & & & & \\
\hline $\operatorname{MELD~(>9~vs.~} \leq 9)$ & $0.756(0.493-1.161)$ & 0.202 & & & & \\
\hline ALBI score 2 vs. 1 & $1.406(0.909-2.175)$ & 0.125 & & & & \\
\hline \multicolumn{7}{|c|}{ HCC } \\
\hline Tumor size (per mm) & $1.053(1.02-1.087)$ & 0.002 & $1.06(1.025-1.097)$ & 0.001 & $1.06(1.014-1.108)$ & 0.01 \\
\hline Tumor size $<20 \mathrm{~mm}$ & $0.615(0.388-0.975)$ & 0.039 & & & & \\
\hline Steatotic HCC & $0.714(0.431-1.184)$ & 0.192 & & & & \\
\hline Dome tumor & $0.736(0.502-1.082)$ & 0.12 & & & & \\
\hline Subcapsular & $0.991(0.713-1.376)$ & 0.955 & & & & \\
\hline Near large vessel & $0.973(0.613-1.544)$ & 0.908 & & & & \\
\hline Near surrounding organ & $1.22(0.696-2.138)$ & 0.488 & & & & \\
\hline \multicolumn{7}{|c|}{ PTA } \\
\hline PTA modality: MWA vs RF & $1.363(0.956-1.943)$ & 0.087 & & & & \\
\hline US vs CT guidance & $1.006(0.712-1.423)$ & 0.973 & & & & \\
\hline Artificial pneumothorax & $1.301(0.569-2.977)$ & 0.533 & & & & \\
\hline Tumor tagging & $0.864(.616-1.212)$ & 0.399 & & & & \\
\hline
\end{tabular}

Abbreviations: HCC, hepatocellular carcinoma; NASH, non-alcoholic steatohepatitis; MELD, model for end-stage liver disease; AFP, alpha fetoprotein; PTA, percutaneous thermal ablation; US, ultrasonography; CT, computed tomography. Significant $p$ values are marked in bold. 


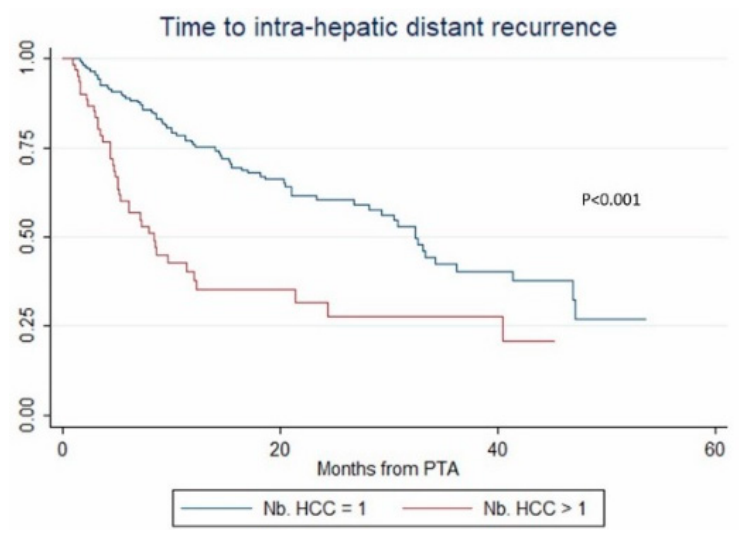

(A)

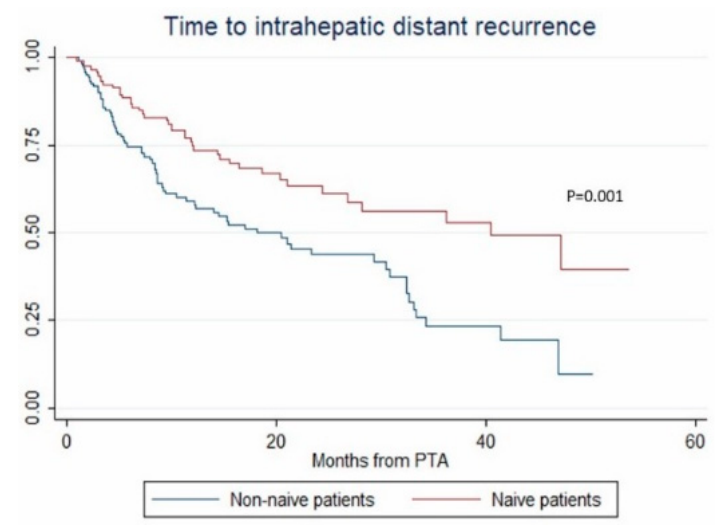

(B)

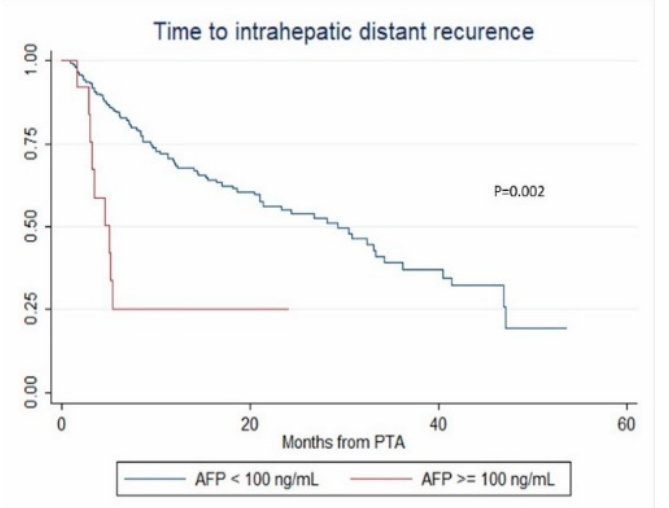

(C)

Figure 3. In patients with uninodular vs. multinodular HCC (A), in treatment-naïve vs. non-treatment naïve patients (B), and in patients with alpha fetoprotein (AFP) $<100 \mathrm{ng} / \mathrm{mL}$ vs. AFP $\geq 100 \mathrm{ng} / \mathrm{mL}$ (C) (Kaplan-Meier analyses).

\subsection{Recurrence-Free Survival}

During the follow-up, HCC relapsed in 52.1\% (124/238) of patients. The 1-year, 2-year and 3-year RFS rates were $60.4 \%$ (95\% CI: 53.5-66.6\%), 46.2\% (95\% CI: $38.6-53.4 \%$ ) and $30.3 \%$ (95\% CI: 21.9-39.1\%), respectively. The median RFS time was 21 months (95\% CI: 15.3-26.8). Over the study period, tumor recurrence was observed in $42.3 \%$ vs. $55.4 \%$ patients with steatotic vs. non-steatotic HCC $(p=0.067)$. LTP was observed after $7.6 \%$ vs. $14.8 \%(p=0.08)$ PTA of steatotic vs. non-steatotic HCC, respectively. IDR was noted in $40.4 \%$ of patients with steatotic and $48.8 \%$ of patients with non-steatotic HCC $(p=0.184)$. In univariate analysis (Table S1), multinodular HCC, treatment-naïve patient, AFP $>100 \mathrm{ng} / \mathrm{mL}$ and steatotic HCC were significantly associated with RFS. In multivariate analysis, multinodular HCC ( $\mathrm{HR}=2.144, p=0.001)$, treatment-naïve patient $(\mathrm{HR}=0.546, p=0.004)$ and AFP $>100 \mathrm{ng} / \mathrm{mL}(\mathrm{HR}=2.437, p=0.013)$ independently predicted RFS (internal validation by bootstrapping). RFS was 7.2 vs. 26.7 months in patients with multinodular HCC vs. uninodular HCC $(p<0.001), 26.8$ vs. 12.3 months in treatment-naïve vs. non-treatment naïve patients $(p<0.001)$ and 21.4 vs. 5.1 months in patients with AFP $<100 \mathrm{ng} / \mathrm{mL}$ vs. AFP $\geq 100 \mathrm{ng} / \mathrm{mL}(p=0.002)$ (Figure 4). 


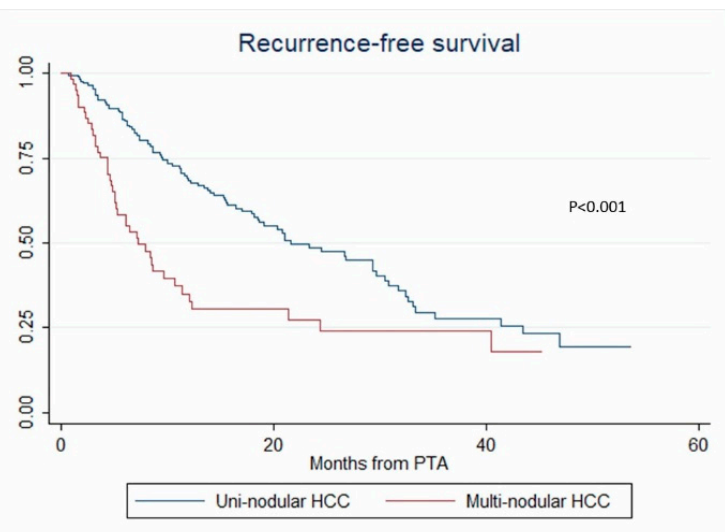

(A)

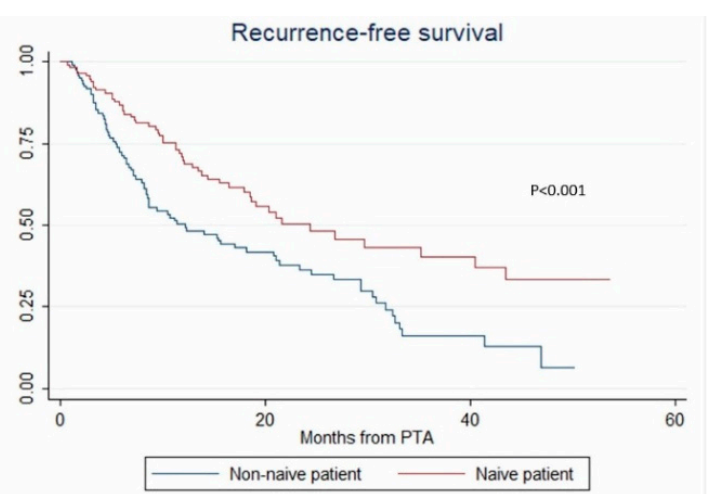

(B)

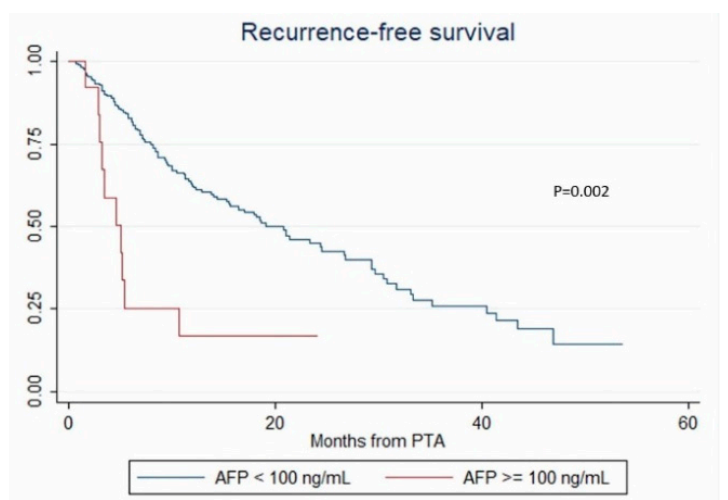

(C)

Figure 4. In patients with uninodular vs. multinodular HCC (A), in treatment naïve vs. non-treatment naïve patients (B), and in patients with $\mathrm{AFP}<100 \mathrm{ng} / \mathrm{mL}$ vs. AFP $\geq 100 \mathrm{ng} / \mathrm{mL}$ (C) (Kaplan-Meier analyses).

\subsection{Overall Survival}

Among the 44 deaths, $36.4 \%$ (16/44) were unrelated to HCC with no evidence of tumor recurrence. The 1-year, 2-year and 3-year OS rate were 98\% (95\% CI: 94.7-99.2\%), 84.3\% (95\% CI: $77.5-89.2 \%)$ and $72.2 \%$ (95\% CI: 62.5-79.8\%), respectively (median not reached).

In univariate analysis, ASA score $>2$, MELD score $>9$, AFP, multinodular HCC, steatotic HCC, $\mathrm{PT}$, bilirubin, creatinine and albumin were significantly associated with OS (Table 4 ). In multivariate analysis, ASA score $>2(\mathrm{HR}=4.273, p=0.011)$, AFP $(\mathrm{HR}=1.002 ; p<0.001)$, multinodular HCC $(\mathrm{HR}=3.939, p=0.003)$ and steatotic HCC $\left(\mathrm{HR}=1.81 \times 10^{-16} ; p<0.001\right)$ independently predicted OS (Figure 5). Only ASA score $>2$, multinodular HCC and steatotic HCC were internally validated as independent predictors of OS (bootstrapping). 
Table 4. Univariate and multivariate Cox regression models to predict overall survival (per patient analysis).

\begin{tabular}{|c|c|c|c|c|c|c|}
\hline \multirow{2}{*}{ Variables } & \multicolumn{2}{|l|}{ Univariate Analysis } & \multicolumn{2}{|l|}{ Multivariate Analysis } & \multicolumn{2}{|c|}{ Bootstrapping (200 Replications) } \\
\hline & Odds Ratio $(95 \% \mathrm{CI})$ & $p$ Value & Odds Ratio $(95 \% \mathrm{CI})$ & $p$ Value & Odds Ratio $(95 \% \mathrm{CI})$ & $p$ Value \\
\hline \multicolumn{7}{|c|}{ Patients } \\
\hline Age & $1.012(0.978-1.046)$ & 0.496 & & & & \\
\hline Sex female vs. male & $0.431(0.150-1.238)$ & 0.118 & & & & \\
\hline ASA (>2 vs. $\leq 2)$ & $2.404(1.248-4.628)$ & 0.009 & $4.273(1.386-13.171)$ & 0.011 & $4.273(1.097-16.646)$ & 0.036 \\
\hline Diabetes & $1.563(0.839-2.912)$ & 0.16 & & & & \\
\hline Metformin treatment & $1.092(0.434-2.752)$ & 0.851 & & & & \\
\hline Treatment-naïve patient & $0.692(0.361-1.326)$ & 0.267 & & & & \\
\hline Local recurrence & $1.016(0.515-2.004)$ & 0.964 & & & & \\
\hline IDR & $1.97(0.988-3.931)$ & 0.054 & & & & \\
\hline \multicolumn{7}{|c|}{ Liver diseases } \\
\hline Cirrhosis & $1.397(0.312-6.258)$ & 0.662 & & & & \\
\hline Child-Pugh (B vs. A) & $1.003(0.094-10.707)$ & 0.998 & & & & \\
\hline \multicolumn{7}{|l|}{ Cause of liver disease (vs. alcohol) } \\
\hline Viral hepatitis B or C & $0.994(0.493-2.005)$ & 0.986 & & & & \\
\hline Hemochromatosis & $0.407(0.062-2.671)$ & 0.349 & & & & \\
\hline Others (including NASH) & $0.537(0.177-1.631)$ & 0.273 & & & & \\
\hline Steatosis & $1.255(0.654-2.406)$ & 0.495 & & & & \\
\hline $\mathrm{AFP} \geq 100 \mathrm{vs}<100 \mathrm{ng} / \mathrm{mL}$ & $4.435(1.456-13.513)$ & 0.009 & & & & \\
\hline AFP (per unit) & $1.0012(1.001-1.003)$ & $<0.001$ & $1.002(1.001-1.003)$ & $<0.001$ & $1.002(0.998-1.006)$ & 0.293 \\
\hline Bilirubin & $1.039(1.002-1.077)$ & 0.038 & & & & \\
\hline Albumin & $0.915(0.86-0.978)$ & 0.009 & & & & \\
\hline Prothrombin time & $0.973(0.949-0.998)$ & 0.031 & & & & \\
\hline Platelet count (per 1000/mm³) & $0.999(0.994-1.003)$ & 0.615 & & & & \\
\hline Creatinine & $1.007(1.002-1.011)$ & 0.003 & & & & \\
\hline $\operatorname{MELD}(>9$ vs. $\leq 9)$ & $2.361(1.253-4.448)$ & 0.008 & $2.014(0.772-5.255)$ & 0.153 & $2.014(0.669-6.063)$ & 0.213 \\
\hline ALBI score 2 vs. 1 & $1.675(0.869-3.23)$ & 0.123 & & & & \\
\hline \multicolumn{7}{|c|}{ HCC } \\
\hline Tumor size (per mm) & $1.023(0.975-1.074)$ & 0.35 & & & & \\
\hline Tumor size $<20 \mathrm{~mm}$ & $1.159(0.498-2.697)$ & 0.731 & & & & \\
\hline $\mathrm{Nb}$. of $\mathrm{HCC}(1 \mathrm{vs} .>1)$ & $1.979(1.003-3.903)$ & 0.049 & $3.939(1.601-9.69)$ & 0.003 & $3.939(1.198-12.947)$ & 0.024 \\
\hline Steatotic HCC & $4.15 \times 10^{-16}\left(2.44 \times 10^{-16}-7.07 \times 10^{-16}\right)$ & $<0.001$ & $1.81 \times 10^{-16}\left(7.96 \times 10^{-17}-4.13 \times 10^{-16}\right)$ & $<0.001$ & $1.81 \times 10^{-16}\left(5.47 \times 10^{-20}-6.02 \times 10^{-13}\right)$ & $<0.001$ \\
\hline Dome tumor & $1.152(0.575-2.307)$ & 0.691 & & & & \\
\hline Subcapsular & $0.813(0.41-1.609)$ & 0.552 & & & & \\
\hline Near large vessel & $1.334(0.683-2.605)$ & 0.399 & & & & \\
\hline Near surrounding organ & $0.404(0.09-1.822)$ & 0.238 & & & & \\
\hline \multicolumn{7}{|c|}{ PTA } \\
\hline PTA modality: MWA vs RF & $1.19(0.845-1.69)$ & 0.08 & & & & \\
\hline US vs CT guidance & $0.797(0.418-1.517)$ & 0.489 & & & & \\
\hline Artificial pneumothorax & $1.301(0.569-2.977)$ & 0.533 & & & & \\
\hline Tumor tagging & $0.774(0.4-1.499)$ & 0.448 & & & & \\
\hline
\end{tabular}

Abbreviations: IDR, intra-hepatic distant recurrence; HCC, hepatocellular carcinoma; NASH, non-alcoholic steatohepatitis; MELD, model for end-stage liver disease; AFP, alpha fetoprotein PTA, percutaneous thermal ablation; US, ultrasonography; CT, computed tomography. Significant $p$ values are marked in bold. 


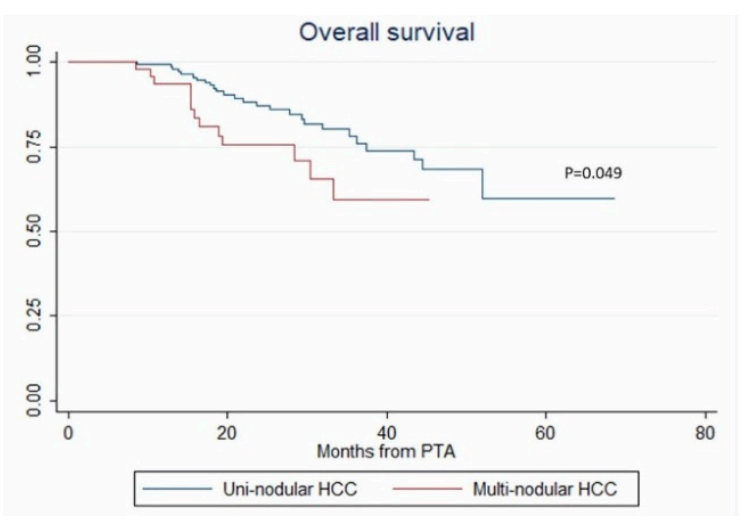

(A)

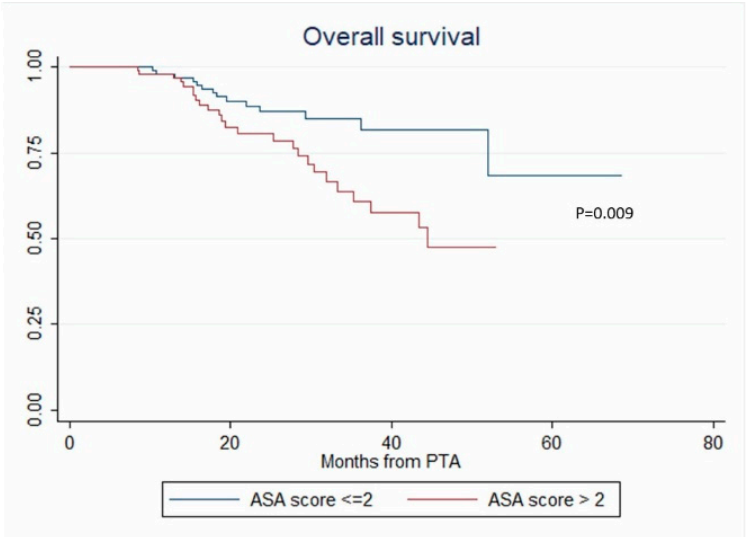

(B)

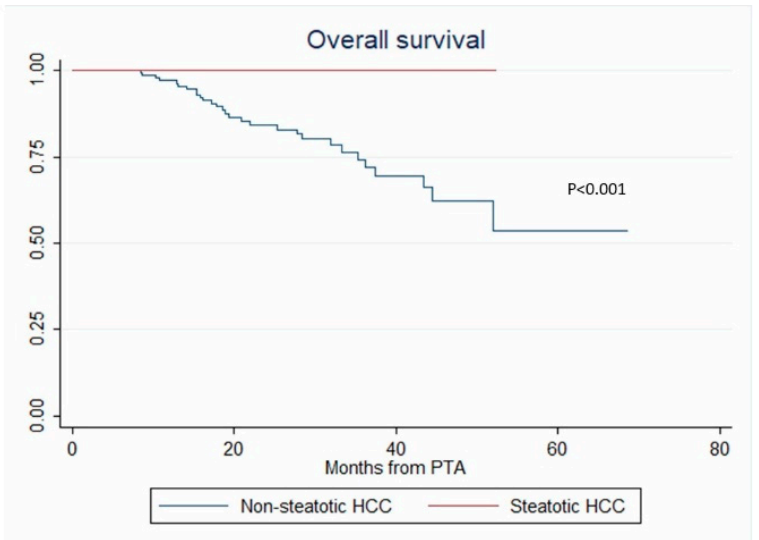

(C)

Figure 5. In patients with uninodular vs. multinodular HCC (A), with ASA score $\leq 2$ vs. $>2$ (B), and with steatotic vs. non-steatotic HCC (C) (Kaplan-Meier analyses).

\section{Discussion}

In this study, we used a multimodal approach to perform PTA of small HCC and investigated 32 variables concerning the patient, liver disease, HCC and PTA technical factors as potential predictors of recurrence and survival. A previous study showed that ultrasonography-guided percutaneous ablation was considered not feasible in $44.5 \%$ of 898 patients during the planning ultrasonography exam, mainly because of inconspicuous HCC (72.8\%) or unsafe needle path (9.5\%) [24]. In a large Korean study (10,334 patients) [3], 56.8\% of RFA for HCC were considered unfeasible based on the planning ultrasonography examination, and $30.3 \%$ of patients $(n=3132)$ received only palliative 
curative treatments. Therefore, using ultrasonography as the only imaging guidance modality for PTA induces a considerable selection bias.

Here we only considered the central HCC location as a technical contraindication and developed a multimodal strategy to address the issues of tumor poor/non-visibility or difficult location. In this study to evaluate this strategy (317 PTAs in 238 patients for $412 \mathrm{HCC}$ ), we reported no treatment-related death, and only $3.6 \%$ of complications, mostly managed conservatively with very limited consequences for the patients. These results are in line with previous series [10,14,25], with the notable exception that we did record any technical mistake (skin burn, gastrointestinal or colon perforation).

Moreover, treatment success (99\%) and LTP (13.3\%) rates compare favorably with recent studies, where LTP ranged between $13.1 \%$ and $20.5 \%$ after PTA of small HCC [3,5,8,13,26,27], especially when considering that we did not exclude patients with non/poor tumor visibility. Interestingly, CT (vs. ultrasonography) guidance was independently associated with LTP, probably because CT is not a real-time imaging modality, thus rendering more challenging the precise puncture of small HCC. We used CT guidance only in the case of poorly-/non-visible tumors by ultrasonography. Conversely, high-risk [11] or difficult tumor locations (dome, subcapsular, near adjacent organs, close to large vessels) and PTA technical factors were not independently associated with LTP, IDR, RFS or OS. Moreover, LTP was frequently $(74.6 \%)$ accessible to repeated PTA sessions $[10,28,29]$, and was not associated with OS, as shown elsewhere [3]. Therefore, multimodal PTA is a valid approach to address challenging PTAs of HCC and avoid any technical contraindication.

Tumor size is usually considered the main LTP predictive factor $[2,10]$. HCC $<2 \mathrm{~cm}$ are considered the best candidates for PTA. Conversely, some debate exists about 2-3 cm HCCs [2]. A recent study reported a 2-fold increased risk of recurrence beyond the Milan criteria in transplantable patients with $>2 \mathrm{~cm} \mathrm{HCC} \mathrm{[13].} \mathrm{In} \mathrm{our} \mathrm{study} \mathrm{(all} \mathrm{HCC}<3 \mathrm{~cm}$ ), tumor size $>2 \mathrm{~cm}$ was associated not only with a $64 \%$ increased risk of LTP, but also a 38\% increased risk of IDR. In addition, even for $\mathrm{HCC}<3 \mathrm{~cm}$, tumor size as a continuous variable was strongly and independently associated with LTP and IDR.

IDR occurred in $47.1 \%$ of patients (i.e., $\approx 3.5$ times more frequent than LTP), in line with other studies $[13,30]$ with comparable follow-up times, and after a median time of only 8.4 months. Distant relapse is due to tumor metastases (more frequent and with poorer prognosis) or de novo carcinogenesis [10,31,32]. Here, we investigated separately IDR occurrence and time-to-IDR. We found that variables reflecting tumor aggressiveness were the only independent predictors of IDR occurrence: AFP $>100 \mathrm{ng} / \mathrm{mL}$ (3-fold increased risk of IDR) and tumor size (IDR risk increased by 6\% per mm of tumor diameter increase). The temporal distribution of IDR after HCC resection [15] suggests that early relapse (within 2-3 years after PTA) is mainly related to tumor features, probably reflecting metastatic spread. In agreement, variables related to tumor aggressiveness (AFP > 100, multinodular HCC) were independently and strongly associated with shorter time-to-IDR (median time-to-IDR $\approx 6$ months) in our series. These findings suggest that IDR was mainly due to metastatic spread rather than de novo carcinogenesis. In the literature, HCC recurrence has been mainly investigated in treatment-naïve patients. By using GEE models, we could incorporate in our analysis all PTAs and take into account that one patient could be treated several times. We found that the risk of IDR occurrence did not vary in treatment-naïve vs. non-treatment naïve patients. Conversely, time-to-IDR in treatment-naïve patients (i.e., first IDR occurrence) was longer (40.4 vs. 18.2 months in non-treatment-naïve patients). Not surprisingly, RFS also was significantly longer in treatment-naïve than non-treatment naïve patients (26.8 vs. 12.3 months, $p<0.001$ ). Although most IDR cases were treated by curative options (mainly repeated PTA) in our series, IDR was close to statistical significance to predict OS. The high IDR rate and accumulating evidence for a metastatic mechanism encourage exploring adjuvant/neoadjuvant strategies targeting tumor growth and metastatic escape in the context of PTA for small HCC.

Not surprisingly, the serum AFP level was strongly and independently associated with both RFS and OS, as shown by other studies [4,7,33-35]. Conversely, limited data are available on the predictive value of the number of HCCs treated by PTA. To our knowledge, only one study compared time-to-IDR and OS in function of the number of nodules treated by PTA [4] and reported HR of 1.36 and 1.28 
for time-to-IDR and OS, respectively, for multinodular (2-3) HCC compared to single tumor. This is in contrast with the strong multi-nodularity effect in our study ( $\mathrm{HR}=3.93$ and 2.67 for time-to-IDR and OS, respectively). A different context (around $85 \%$ of viral infections, only $14.5 \%$ of high alcohol consumption) and more advanced HCC ( $29.7 \%$ of patients with $>3 \mathrm{~cm}$ or $>3$ HCC nodules) [4] might explain the weaker effect of multi-nodularity in this previous study.

Surprisingly, none of the usual prognostic factors of liver disease was independently associated with RFS or OS. MELD score $>9$, high bilirubin level and low albumin and PT values were associated with shorter OS in the univariate analysis. However, only a MELD score $>9$ was included in the multivariate analysis (i) because of colinearity with other covariates, (ii) to prevent overfitting given the low number of events (i.e., 44 deaths) and (iii) because it had the strongest association with OS among these variables in the univariate analysis. The relatively short follow-up and the strong weight of tumor aggressiveness variables (AFP, and nodule number), or ASA score in survival models could also explain why the MELD score did not independently predict survival.

One of most striking results in this study is the independent predictive value of steatotic HCC for OS. Literature data on steatotic HCCs are very limited. In 2000, Kutami et al. [36] found that fat content in HCC at pathologic examination (51/260 HCC) was more frequent in small HCC $(1.1-1.5 \mathrm{~cm})$ and in the well-differentiated subtype. More recently, Chan et al. [37] showed that steatotic HCC were associated with higher metabolic risk (diabetes, arterial hypertension, steatohepatitis), smaller tumor size, lower frequency of major vessel and microvascular invasion, earlier tumor stages and lower serum AFP. In 2010, Salamao et al. first described steatohepatitic HCC, characterized by inflammatory changes, cell ballooning, peri-cellular fibrosis and Mallory-Denk bodies [38]. This variant is frequently observed in a nonalcoholic steatohepatitis (NASH) context. Analysis of the molecular and genetic alterations of steatohepatitic HCCs showed that they belong to the G4 sub-class (known to have a gene expression profile similar to that of nontumoral liver) with more favorable prognosis, they very rarely harbor the activated Wnt/beta-catenin pathway, and they lack CTNNB1, TP53 and TERT promoter mutations [39]. In 2016, Chan et al. demonstrated that steatohepatitic HCC is a subset of steatotic HCC [37], and showed that steatotic HCC was associated with late tumor relapse after surgery [37]. In our series, RFS was longer in patients with steatotic HCC (univariate analysis), whereas LTP, time-to-LTP, IDR and time-to-IDR were not different in patients with steatotic vs. non-steatotic HCC. Again, ethnicity (Asian vs. European patients) and treatment (surgical vs. ablation) strongly differ between the study by Chan et al. and our work. Nevertheless, steatotic HCC strongly and independently predicted longer OS and none of our patients with steatotic HCC died during the follow-up. In our study, $17.2 \%$ of HCC were steatotic, like in previous series $[36,37]$ where this definition was based on pathology findings. Chemical-shift imaging takes advantage of the difference in resonance frequency between water and fat, and is part of routine MRI protocols. It detects intratumoral steatosis with $100 \%$ specificity $[40,41]$, allowing the noninvasive diagnosis of steatotic HCC. Accumulating evidence of a more favorable outcome strongly encourages researchers to further analyze the genomic profile of steatotic HCCs [42].

Several limitations to our study must be acknowledged. First, this is a retrospective, monocentric study, although data came from a prospectively maintained database with a strict imaging follow-up policy. We chose to include treatment-naïve and non-treatment naïve patients to explore this variable with GEE models, because a patient can be treated several times. The selection bias inherent to retrospective studies was very limited, because we included only patients with $\mathrm{HCC}$ nodules $<3 \mathrm{~cm}$ that were not centrally-located, owing to the multimodal interventional suite. Second, the median follow-up was relatively short (27.1 months). This explains the relatively low number of death events, and the limited evaluation of late HCC recurrences. Third, we could not retrospectively collect reliable information regarding abstinence, which could have influenced the overall survival of patients with alcohol-induced cirrhosis.

Then, our results need to be validated in an external series, although we systematically used bootstrapping as internal validation and to prevent overfitting [43]. Finally, like in most HCC series, the 
very low proportion of patients with liver biopsy did not allow exploring the tumor molecular/genetic features, especially in the case of steatotic HCC.

\section{Conclusions}

This study shows that multimodal PTA is safe and effective for treating small HCC, whatever the technical difficulty to see or to reach the tumor, and even in presumed challenging locations. CT guidance carries an increased risk of LTP, but was not associated with any survival endpoint, justifying its use as second-line guidance modality. IDR remains the most frequent event during the follow-up of patients treated by PTA for small HCC, and was associated with both tumor size and AFP, suggesting a metastatic mechanism. Besides AFP association with LTP, IDR, RFS and OS, treatment-naïve patients had longer RFS than non-treatment naïve patients, and multi-nodularity was associated with shorter RFS and OS. Finally, steatotic HCC independently predicted longer OS. This specific HCC variant, which can be identified in the pre-treatment MRI, needs to be further explored.

Supplementary Materials: The following are available online at http://www.mdpi.com/2072-6694/12/2/313/s1, Figure S1: In-phase (A) and opposed-phase (B) chemical-shift imaging showing signal intensity loss on the opposed-phase image (arrow) in a typical steatotic HCC, Figure S2 Intra-arterial injection of lipiodol to tag a HCC invisible by ultrasonography (A). Then, the HCC becomes hyperdense and a microwave ablation needle is inserted under CT-guidance (B and C), Figure S3 After intra-arterial injection of lipiodol to tag a HCC located under the liver dome (thus invisible by ultrasonography), pneumothorax was artificially induced with $\mathrm{CO}_{2}$ using a Veress needle (A) and the radiofrequency-ablation needle was inserted through the extrapulmonary transthoracic transdiaphragmatic route (B), Figure S4 Three radiofrequency ablation needles were inserted in a HCC nodule located close to the middle hepatic vein (A). Then, an $11 \mathrm{~mm}$-balloon was inflated to stop the blood flow in the middle hepatic vein in order to prevent the heat sink effect (B). Table S1: Table S1. Univariate and multivariate Cox regression models to predict recurrence-free survival (per patient analysis), Table S2. Complications observed after 412 PTA sessions.

Author Contributions: Conceptualization, B.G.; Methodology, B.G. and S.A.-G.; Validation, M.H. and B.G.; Formal Analysis, M.H. and S.A-G. and B.G.; Investigation, all authors: Writing-Original Draft Preparation, M.H. and B.G.; Writing-Review \& Editing, all authors; Project Administration, B.G. B.G. contributed to the study design; M.H., C.C., L.P., C.G., V.S., C.A., S.J., G.-P.P., E.A., B.G. performed the research; M.H., C.C., L.P., S.A.-G., C.G., V.S., C.A., S.J., G.-P.P., E.A., B.G. collected and analysed the data. All authors have read and agreed to the published version of the manuscript.

Funding: This research received no external funding.

Acknowledgments: The authors thank Elisabetta Andermarcher for revising the English.

Conflicts of Interest: The authors declare no conflict of interest.

\section{References}

1. Global Burden of Disease Cancer, C.; Fitzmaurice, C.; Abate, D.; Abbasi, N.; Abbastabar, H.; Abd-Allah, F.; Abdel-Rahman, O.; Abdelalim, A.; Abdoli, A.; Abdollahpour, I.; et al. Global, Regional, and National Cancer Incidence, Mortality, Years of Life Lost, Years Lived With Disability, and Disability-Adjusted Life-Years for 29 Cancer Groups, 1990 to 2017: A Systematic Analysis for the Global Burden of Disease Study. JAMA Oncol. 2019. [CrossRef]

2. European Association for the Study of the Liver. EASL Clinical Practice Guidelines: Management of hepatocellular carcinoma. J. Hepatol. 2018. [CrossRef]

3. Kim, Y.-S.; Lim, H.K.; Rhim, H.; Lee, M.W.; Choi, D.; Lee, W.J.; Paik, S.W.; Koh, K.C.; Lee, J.H.; Choi, M.S.; et al. Ten-year outcomes of percutaneous radiofrequency ablation as first-line therapy of early hepatocellular carcinoma: Analysis of prognostic factors. J. Hepatol. 2013, 58, 89-97. [CrossRef] [PubMed]

4. Shiina, S.; Tateishi, R.; Arano, T.; Uchino, K.; Enooku, K.; Nakagawa, H.; Asaoka, Y.; Sato, T.; Masuzaki, R.; Kondo, Y.; et al. Radiofrequency ablation for hepatocellular carcinoma: 10-year outcome and prognostic factors. Am. J. Gastroenterol. 2012, 107, 569-577. [CrossRef] [PubMed]

5. Kang, T.W.; Kim, J.M.; Rhim, H.; Lee, M.W.; Kim, Y.S.; Lim, H.K.; Choi, D.; Song, K.D.; Kwon, C.H.; Joh, J.W.; et al. Small Hepatocellular Carcinoma: Radiofrequency Ablation versus Nonanatomic Resection-Propensity Score Analyses of Long-term Outcomes. Radiology 2015, 275, 908-919. [CrossRef] [PubMed] 
6. Hocquelet, A.; Balageas, P.; Laurent, C.; Blanc, J.F.; Frulio, N.; Salut, C.; Cassinotto, C.; Saric, J.; Possenti, L.; Bernard, P.H.; et al. Radiofrequency ablation versus surgical resection for hepatocellular carcinoma within the Milan criteria: A study of 281 Western patients. Int. J. Hyperthermia 2015, 31, 749-757. [CrossRef] [PubMed]

7. N'Kontchou, G.; Mahamoudi, A.; Aout, M.; Ganne-Carrié, N.; Grando, V.; Coderc, E.; Vicaut, E.; Trinchet, J.C.; Sellier, N.; Beaugrand, M.; et al. Radiofrequency ablation of hepatocellular carcinoma: Long-term results and prognostic factors in 235 Western patients with cirrhosis. Hepatology 2009, 50, 1475-1483. [CrossRef] [PubMed]

8. Hocquelet, A.; Aubé, C.; Rode, A.; Cartier, V.; Sutter, O.; Manichon, A.F.; Boursier, J.; N’kontchou, G.; Merle, P.; Blanc, J.-F.; et al. Comparison of no-touch multi-bipolar vs. monopolar radiofrequency ablation for small HCC. J. Hepatol. 2017, 66, 67-74. [CrossRef]

9. Cui, J.; de Klerk, N.; Abramson, M.; Del Monaco, A.; Benke, G.; Dennekamp, M.; Musk, A.W.; Sim, M. Fractional polynomials and model selection in generalized estimating equations analysis, with an application to a longitudinal epidemiologic study in Australia. Am. J. Epidemiol. 2009, 169, 113-121. [CrossRef]

10. Nault, J.C.; Sutter, O.; Nahon, P.; Ganne-Carrie, N.; Seror, O. Percutaneous treatment of hepatocellular carcinoma: State of the art and innovations. J. Hepatol. 2018, 68, 783-797. [CrossRef]

11. Teratani, T.; Yoshida, H.; Shiina, S.; Obi, S.; Sato, S.; Tateishi, R.; Mine, N.; Kondo, Y.; Kawabe, T.; Omata, M. Radiofrequency ablation for hepatocellular carcinoma in so-called high-risk locations. Hepatology 2006, 43, 1101-1108. [CrossRef] [PubMed]

12. Kim, J.-E.; Kim, Y.-S.; Rhim, H.; Lim, H.K.; Lee, M.W.; Choi, D.; Shin, S.W.; Cho, S.K. Outcomes of patients with hepatocellular carcinoma referred for percutaneous radiofrequency ablation at a tertiary center: Analysis focused on the feasibility with the use of ultrasonography guidance. Eur. J. Radiol. 2011, 79, e80-e84. [CrossRef] [PubMed]

13. Doyle, A.; Gorgen, A.; Muaddi, H.; Aravinthan, A.D.; Issachar, A.; Mironov, O.; Zhang, W.; Kachura, J.; Beecroft, R.; Cleary, S.P.; et al. Outcomes of radiofrequency ablation as first-line therapy for hepatocellular carcinoma less than $3 \mathrm{~cm}$ in potentially transplantable patients. J. Hepatol. 2019, 70, 866-873. [CrossRef] [PubMed]

14. Livraghi, T.; Meloni, F.; Di Stasi, M.; Rolle, E.; Solbiati, L.; Tinelli, C.; Rossi, S. Sustained complete response and complications rates after radiofrequency ablation of very early hepatocellular carcinoma in cirrhosis: Is resection still the treatment of choice? Hepatology 2008, 47, 82-89. [CrossRef]

15. Imamura, H.; Matsuyama, Y.; Tanaka, E.; Ohkubo, T.; Hasegawa, K.; Miyagawa, S.; Sugawara, Y.; Minagawa, M.; Takayama, T.; Kawasaki, S.; et al. Risk factors contributing to early and late phase intrahepatic recurrence of hepatocellular carcinoma after hepatectomy. J. Hepatol. 2003, 38, 200-207. [CrossRef]

16. Okuwaki, Y.; Nakazawa, T.; Kokubu, S.; Hidaka, H.; Tanaka, Y.; Takada, J.; Watanabe, M.; Shibuya, A.; Minamino, T.; Saigenji, K. Repeat radiofrequency ablation provides survival benefit in patients with intrahepatic distant recurrence of hepatocellular carcinoma. Am. J. Gastroenterol. 2009, 104, 2747-2753. [CrossRef]

17. Hermida, M.; Cassinotto, C.; Piron, L.; Assenat, E.; Pageaux, G.P.; Escal, L.; Pierredon-Foulongne, M.A.; Verzilli, D.; Jaber, S.; Guiu, B. Percutaneous thermal ablation of hepatocellular carcinomas located in the hepatic dome using artificial carbon dioxide pneumothorax: Retrospective evaluation of safety and efficacy. Int. J. Hyperthermia 2018, 35, 90-96. [CrossRef]

18. De Baère, T.; Dromain, C.; Lapeyre, M.; Briggs, P.; Duret, J.S.; Hakime, A.; Boige, V.; Ducreux, M. Artificially induced pneumothorax for percutaneous transthoracic radiofrequency ablation of tumors in the hepatic dome: Initial experience. Radiology 2005, 236, 666-670. [CrossRef]

19. Kariya, S.; Tanigawa, N.; Kojima, H.; Komemushi, A.; Shomura, Y.; Ueno, Y.; Shiraishi, T.; Sawada, S. Radiofrequency ablation combined with $\mathrm{CO} 2$ injection for treatment of retroperitoneal tumor: Protecting surrounding organs against thermal injury. AJR Am. J. Roentgenol. 2005, 185, 890-893. [CrossRef]

20. De Baere, T.; Deschamps, F.; Briggs, P.; Dromain, C.; Boige, V.; Hechelhammer, L.; Abdel-Rehim, M.; Aupérin, A.; Goere, D.; Elias, D. Hepatic malignancies: Percutaneous radiofrequency ablation during percutaneous portal or hepatic vein occlusion. Radiology 2008, 248, 1056-1066. [CrossRef]

21. Cardella, J.F.; Kundu, S.; Miller, D.L.; Millward, S.F.; Sacks, D.; Radiology, S.O.I. Society of Interventional Radiology clinical practice guidelines. J. Vasc. Interven. Radiol. 2009, 20, S189-S191. [CrossRef] [PubMed]

22. Ahmed, M.; Solbiati, L.; Brace, C.L.; Breen, D.J.; Callstrom, M.R.; Charboneau, J.W.; Chen, M.-H.; Choi, B.I.; de Baère, T.; Dodd, G.D.; et al. Image-guided tumor ablation: Standardization of terminology and reporting criteria-a 10-year update. J. Vasc. Interven. Radiol. 2014, 25. [CrossRef] 
23. Pan, W. Akaike's information criterion in generalized estimating equations. Biometrics 2001, 57, $120-125$. [CrossRef] [PubMed]

24. Kim, P.N.; Choi, D.; Rhim, H.; Rha, S.E.; Hong, H.P.; Lee, J.; Choi, J.I.; Kim, J.W.; Seo, J.W.; Lee, E.J.; et al. Planning ultrasound for percutaneous radiofrequency ablation to treat small $(\leq 3 \mathrm{~cm})$ hepatocellular carcinomas detected on computed tomography or magnetic resonance imaging: A multicenter prospective study to assess factors affecting ultrasound visibility. J. Vasc. Interven. Radiol. 2012, 23, 627-634. [CrossRef]

25. Kasugai, H.; Osaki, Y.; Oka, H.; Kudo, M.; Seki, T.; Osaka Liver Cancer Study, G. Severe complications of radiofrequency ablation therapy for hepatocellular carcinoma: An analysis of 3,891 ablations in 2614 patients. Oncology 2007, 72 (Suppl. 1), 72-75. [CrossRef]

26. Kang, T.W.; Lim, H.K.; Lee, M.W.; Kim, Y.-S.; Rhim, H.; Lee, W.J.; Paik, Y.H.; Kim, M.J.; Ahn, J.H. Long-term Therapeutic Outcomes of Radiofrequency Ablation for Subcapsular versus Nonsubcapsular Hepatocellular Carcinoma: A Propensity Score Matched Study. Radiology 2016, 280, 300-312. [CrossRef]

27. Pompili, M.; Saviano, A.; de Matthaeis, N.; Cucchetti, A.; Ardito, F.; Federico, B.; Brunello, F.; Pinna, A.D.; Giorgio, A.; Giulini, S.M.; et al. Long-term effectiveness of resection and radiofrequency ablation for single hepatocellular carcinoma $\leq 3 \mathrm{~cm}$. Results of a multicenter Italian survey. J. Hepatol. 2013, 59, 89-97. [CrossRef]

28. Rossi, S.; Ravetta, V.; Rosa, L.; Ghittoni, G.; Viera, F.T.; Garbagnati, F.; Silini, E.M.; Dionigi, P.; Calliada, F.; Quaretti, P.; et al. Repeated radiofrequency ablation for management of patients with cirrhosis with small hepatocellular carcinomas: A long-term cohort study. Hepatology 2011, 53, 136-147. [CrossRef]

29. Tateishi, R.; Shiina, S.; Teratani, T.; Obi, S.; Sato, S.; Koike, Y.; Fujishima, T.; Yoshida, H.; Kawabe, T.; Omata, M. Percutaneous radiofrequency ablation for hepatocellular carcinoma. An analysis of 1000 cases. Cancer 2005, 103, 1201-1209. [CrossRef]

30. Okuwaki, Y.; Nakazawa, T.; Shibuya, A.; Ono, K.; Hidaka, H.; Watanabe, M.; Kokubu, S.; Saigenji, K. Intrahepatic distant recurrence after radiofrequency ablation for a single small hepatocellular carcinoma: Risk factors and patterns. J. Gastroenterol. 2008, 43, 71-78. [CrossRef]

31. Miao, R.; Luo, H.; Zhou, H.; Li, G.; Bu, D.; Yang, X.; Zhao, X.; Zhang, H.; Liu, S.; Zhong, Y.; et al. Identification of prognostic biomarkers in hepatitis $B$ virus-related hepatocellular carcinoma and stratification by integrative multi-omics analysis. J. Hepatol. 2014, 61, 840-849. [CrossRef] [PubMed]

32. Wang, B.; Xia, C.Y.; Lau, W.Y.; Lu, X.Y.; Dong, H.; Yu, W.L.; Jin, G.Z.; Cong, W.M.; Wu, M.C. Determination of clonal origin of recurrent hepatocellular carcinoma for personalized therapy and outcomes evaluation: A new strategy for hepatic surgery. J. Am. Coll Surg. 2013, 217, 1054-1062. [CrossRef]

33. Choi, D.; Lim, H.K.; Rhim, H.; Kim, Y.-S.; Lee, W.J.; Paik, S.W.; Koh, K.C.; Lee, J.H.; Choi, M.S.; Yoo, B.C. Percutaneous radiofrequency ablation for early-stage hepatocellular carcinoma as a first-line treatment: Long-term results and prognostic factors in a large single-institution series. European Radiol. 2007, 17, 684-692. [CrossRef] [PubMed]

34. Lee, D.H.; Lee, J.M.; Lee, J.Y.; Kim, S.H.; Yoon, J.H.; Kim, Y.J.; Han, J.K.; Choi, B.I. Radiofrequency ablation of hepatocellular carcinoma as first-line treatment: Long-term results and prognostic factors in 162 patients with cirrhosis. Radiology 2014, 270, 900-909. [CrossRef] [PubMed]

35. Kim, Y.-S.; Rhim, H.; Cho, O.K.; Koh, B.H.; Kim, Y. Intrahepatic recurrence after percutaneous radiofrequency ablation of hepatocellular carcinoma: Analysis of the pattern and risk factors. Eur. J. Radiol. 2006, 59, 432-441. [CrossRef] [PubMed]

36. Kutami, R.; Nakashima, Y.; Nakashima, O.; Shiota, K.; Kojiro, M. Pathomorphologic study on the mechanism of fatty change in small hepatocellular carcinoma of humans. J. Hepatol. 2000, 33, 282-289. [CrossRef]

37. Chan, A.W.H.; Yu, S.; Yu, Y.-H.; Tong, J.H.M.; Wang, L.; Tin, E.K.Y.; Chong, C.C.N.; Chan, S.L.; Wong, G.L.H.; Wong, V.W.S.; et al. Steatotic hepatocellular carcinoma: A variant associated with metabolic factors and late tumour relapse. Histopathology 2016, 69, 971-984. [CrossRef]

38. Salomao, M.; Yu, W.M.; Brown, R.S.; Emond, J.C.; Lefkowitch, J.H. Steatohepatitic hepatocellular carcinoma (SH-HCC): A distinctive histological variant of HCC in hepatitis $\mathrm{C}$ virus-related cirrhosis with associated NAFLD/NASH. Am. J. Surg. Pathol. 2010, 34, 1630-1636. [CrossRef]

39. Calderaro, J.; Ziol, M.; Paradis, V.; Zucman-Rossi, J. Molecular and histological correlations in liver cancer. J. Hepatol. 2019, 71, 616-630. [CrossRef]

40. Guiu, B.; Petit, J.M.; Loffroy, R.; Ben Salem, D.; Aho, S.; Masson, D.; Hillon, P.; Krause, D.; Cercueil, J.P. Quantification of liver fat content: Comparison of triple-echo chemical shift gradient-echo imaging and in vivo proton MR spectroscopy. Radiology 2009, 250, 95-102. [CrossRef] 
41. Min, J.H.; Kim, Y.K.; Lim, S.; Jeong, W.K.; Choi, D.; Lee, W.J. Prediction of microvascular invasion of hepatocellular carcinomas with gadoxetic acid-enhanced MR imaging: Impact of intra-tumoral fat detected on chemical-shift images. Eur. J. Radiol. 2015, 84, 1036-1043. [CrossRef]

42. Nault, J.C.; Martin, Y.; Caruso, S.; Hirsch, T.Z.; Bayard, Q.; Calderaro, J. Clinical Impact of Genomic Diversity From Early to Advanced Hepatocellular Carcinoma. Hepatology 2019. [CrossRef]

43. Efron, B.; Tibshirani, R.J. An Introduction to the Bootstrap (Monographs on Statistics and Applied Probability); Chapman \& Hall/CRC: Boca Raton, FL, USA, 1993.

(C) 2020 by the authors. Licensee MDPI, Basel, Switzerland. This article is an open access article distributed under the terms and conditions of the Creative Commons Attribution (CC BY) license (http://creativecommons.org/licenses/by/4.0/). 